\title{
Blockchain and Artificial Intelligence Applications
}

\author{
Guanqiu Qi ${ }^{1}$ and Zhiqin Zhu ${ }^{2}$ \\ ${ }^{1}$ Computer Information Systems Department, State University of New York at Buffalo State, Buffalo, NY 14222, USA \\ ${ }^{2}$ College of Automation, Chongqing University of Posts and Telecommunications, Chongqing 400065, China
}

(Received 16 April 2021; Revised 16 April 2021; Accepted 19 April 2021; Published online 30 April 2021)

The emergence and popularity of blockchain, distributed ledger technology distributed computing, and network security and trust techniques are significantly changing the operation and management of computing and communication systems, as these techniques have the potential to disrupt any domain involving coordination among autonomous resources without trusted third parties. These techniques and their applications include finance and payments (e.g., Facebook Libra), but also networks (e.g., power grids or telecom networks), computing (e.g., brokering of edge resources), Internet of Things (e.g., supply chain or industry 4.0), and service platforms (e.g., identity management). The market capitalization, investor appetite, and institutional coverage for cryptocurrency (as well as bitcoin and blockchain) have all jumped exponentially. The total market capitalization of the cryptocurrency market has significantly increased in the past three years. The applications of blockchain exhibit a variety of complicated problems and new requirements, which brings more open issues and challenges for artificial intelligence (AI) and related research.

In this special issue of the Journal of Artificial Intelligence and Technology (JAIT), we are pleased to publish seven research papers that open up the latest research on blockchain and other AI applications. We have undergone a rigorous peer review and revision process to ensure high-quality and scientific significance. The following paragraphs describe the contents of the seven papers. The first two papers study the blockchain technologies and their applications in joint governance of public health and security incidents and in security authentication. The remaining papers discuss AI applications.

The paper "An innovative mechanism of blockchain technology on joint governance model," authored by Huawei Zhao and Ruzhi Xu, studies the blockchain model for joint governance of public health and security incidents. It focuses on the multiagent collaborative prevention and control governance model, which provides a new opportunity for model innovation in data governance and cooperative governance.

The paper "Cross-heterogeneous domain authentication scheme based on blockchain," authored by Jing Liu, Yixin Liu, Yingxu Lai, Rongchen Li, Siyu Wu, and Sami Mian, proposes and establishes a reliable cross-domain authentication scheme by using blockchain techniques, which solves the issues occurring during crossing heterogeneous authentication domains and guaranteeing the security of the certification process.

The paper "Research on intelligent control system of thermal print head based on field programmable gate array," authored by
Yang $\mathrm{Xu}$, Sheng Wang, Ying Peng, and Matthew Haner, proposes a field-programmable gate array. It is based on a thermal print head heating control method to improve the stability of the thermal print head during printing, and it solves the inefficiency of the traditional single-chip microcomputer control of the thermal print head heating method.

The paper "Video-based person reidentification based on distributed cloud computing," authored by Chengyan Zhong, Xiaoyu Jiang, and Guanqiu Qi, applies distributed cloud computing method to perform person reidentification (re-ID) tasks, which solves the scalability issue of person re-ID and achieves the realtime processing of person re-ID tasks.

The paper "Intelligent voice instructor-assistant system for collaborative and interactive classes," authored by Matthew Baker, Xiaohui $\mathrm{Hu}$, Gennaro De Luca, and Yinong Chen, develops a prototype of an Intelligent Voice Instructor-Assistant System (IVIAS) for supporting large classes, in which Amazon Web Services, Alexa Voice Services, and self-developed services are used. The performance of IVIAS grows through learning and improving when more and more questions are asked and answered.

The paper "Estimation of low nutrients in tomato crops through the analysis of leaf images using machine learning," authored by Hiram Ponce, Claudio Cevallos, Ricardo Espinosa, and Sebastian Gutierrez, proposes a novel convolutional neural network (CNN)-based classifier, namely, $\mathrm{CNN}+\mathrm{AHN}$, for estimating low nutrients in tomato crops using an image of the tomato leaves. The proposed CNN+AHN incorporates a set of convolutional layers as the feature extraction part, and a supervised learning method called artificial hydrocarbon network (AHN) as the dense layer.

The paper "Deep neural network-based speaker-aware information logging for augmentative and alternative communication," authored by Gang Hu, Szu-Han Kay Chen, and Neal Mazur, presents a solution using a deep neural network-based visual analysis approach to process videos to detect different augmentative and alternative communication (AAC) users in practice sessions, which has significant potential to improve the validity of data logs and ultimately to enhance AAC outcome measures.

The guest editors express great appreciation to all the reviewers, the JAIT Editor-in-Chief, Dr. Yinong Chen, the journal manager, and the whole journal staff for their invaluable support in setting up this special issue. 